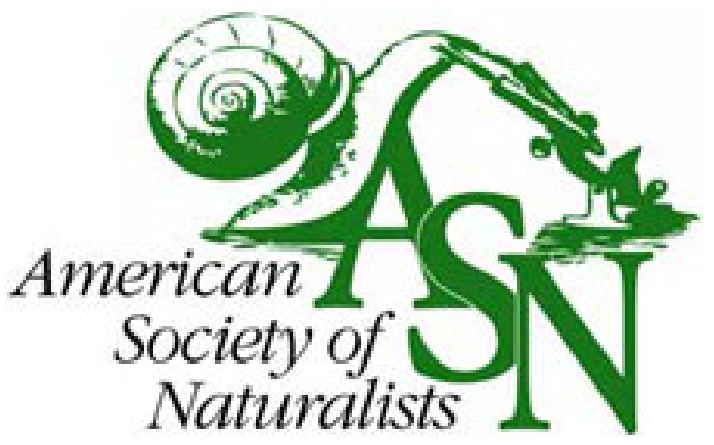

The University of Chicago

Rapid Independent Trait Evolution despite a Strong Pleiotropic Genetic Correlation.

Author(s): Jeffrey K. Conner, Keith Karoly, Christy Stewart, Vanessa A. Koelling, Heather F. Sahli, and Frank H. Shaw

Reviewed work(s):

Source: The American Naturalist, Vol. 178, No. 4 (October 2011), pp. 429-441

Published by: The University of Chicago Press for The American Society of Naturalists

Stable URL: http://www.jstor.org/stable/10.1086/661907

Accessed: $31 / 10 / 2012$ 16:24

Your use of the JSTOR archive indicates your acceptance of the Terms \& Conditions of Use, available at

http://www.jstor.org/page/info/about/policies/terms.jsp

JSTOR is a not-for-profit service that helps scholars, researchers, and students discover, use, and build upon a wide range of content in a trusted digital archive. We use information technology and tools to increase productivity and facilitate new forms of scholarship. For more information about JSTOR, please contact support@jstor.org. 


\title{
Rapid Independent Trait Evolution despite a Strong Pleiotropic Genetic Correlation
}

\author{
Jeffrey K. Conner, ${ }^{1, \star}$ Keith Karoly, ${ }^{2}$ Christy Stewart, ${ }^{1, \dagger}$ Vanessa A. Koelling, ${ }^{2, \ddagger}$ \\ Heather F. Sahli, ${ }^{1, \$}$ and Frank H. Shaw ${ }^{3}$
}

1. Kellogg Biological Station and Department of Plant Biology, Michigan State University, Hickory Corners, Michigan 49060; 2. Biology Department, Reed College, Portland, Oregon 97202; 3. Department of Mathematics, Hamline University, St. Paul, Minnesota 55108

Submitted November 2, 2010; Accepted June 8, 2011; Electronically published August 24, 2011

Dryad data: http://dx.doi.org/10.5061/dryad.41pn9.

ABSTRACT: Genetic correlations are the most commonly studied of all potential constraints on adaptive evolution. We present a comprehensive test of constraints caused by genetic correlation, comparing empirical results to predictions from theory. The additive genetic correlation between the filament and the corolla tube in wild radish flowers is very high in magnitude, is estimated with good precision $(0.85 \pm 0.06)$, and is caused by pleiotropy. Thus, evolutionary changes in the relative lengths of these two traits should be constrained. Still, artificial selection produced rapid evolution of these traits in opposite directions, so that in one replicate relative to controls, the difference between them increased by six standard deviations in only nine generations. This would result in a $54 \%$ increase in relative fitness on the basis of a previous estimate of natural selection in this population, and it would produce the phenotypes found in the most extreme species in the family Brassicaceae in less than 100 generations. These responses were within theoretical expectations and were much slower than if the genetic correlation was zero; thus, there was evidence for constraint. These results, coupled with comparable results from other species, show that evolution can be rapid despite the constraints caused by genetic correlations.

Keywords: genetic correlation, evolutionary constraint, rapid evolution, artificial selection, radish.

\section{Introduction}

Constraints on adaptive evolution, defined as anything that slows or prevents the attainment of an optimally adapted phenotype, have been a topic of major interest since the publication of Gould and Lewontin's (1979) study. In spite

* Corresponding author; e-mail connerj@msu.edu.

${ }^{\dagger}$ Present address: Department of Entomology, University of Wisconsin, Madison, Wisconsin 53706.

¥ Present address: Department of Ecology and Evolutionary Biology, University of Kansas, Lawrence, Kansas 66045.

$\S$ Present address: Department of Biology, Shippensburg University, Shippensburg, Pennsylvania 17257.

Am. Nat. 2011. Vol. 178, pp. 429-441. () 2011 by The University of Chicago. 0003-0147/2011/17804-52591\$15.00. All rights reserved. DOI: $10.1086 / 661907$ of a large number of studies addressing constraints (e.g., O'Neil and Schmitt 1993; Futuyma et al. 1995; MitchellOlds 1996; Pilson 1996; Begin and Roff 2003; Frankino et al. 2005; Ashman and Majetic 2006), much confusion and controversy remains over definitions, the possible mechanistic bases of constraints, and the relative importance of different mechanisms (Gould 1989; Antonovics and van Tienderen 1991; Arnold 1992; Getty 2000; Pigliucci and Kaplan 2000). Genetic correlations among traits have often been invoked as a cause of constraint (e.g., Maynard Smith et al. 1985; Clark 1987; Arnold 1992; Futuyma 2010), and many estimates of genetic correlations have been undertaken in the last two decades, with the goal of understanding constraints (e.g., Berenbaum et al. 1986; Conner and Via 1992; Etterson and Shaw 2001; Delph et al. 2004; Caruso et al. 2005).

The theoretical underpinnings for the idea that genetic correlations cause constraint were developed some time ago (Dickerson 1955; Lande 1976). The general idea is simple: a population evolves in multivariate trait space at a rate that depends on the magnitude and the direction of the vector of selection and the amount of genetic variance possessed by the population in that direction. Thus, in two dimensions, if a genetic correlation is 1 or -1 , no evolution can take place in the direction perpendicular to the major axis of the correlation because there is no genetic variance in that direction (Via and Lande 1985; Pease and Bull 1988). This case, where a genetic correlation prevents the population from reaching the multivariate optimum, is called an absolute constraint (Mezey and Houle 2005), and it has also been predicted to occur with a correlation of $<1$ if there are other correlated traits under stabilizing selection (Björklund 1996). For genetic correlations with a magnitude of $<1$, there is little theoretical doubt that selection perpendicular to the axis of a correlation (the arrows in fig. $1 \mathrm{~A}$ ) will result in a slower response than the selection parallel to it, because there is maximal genetic 


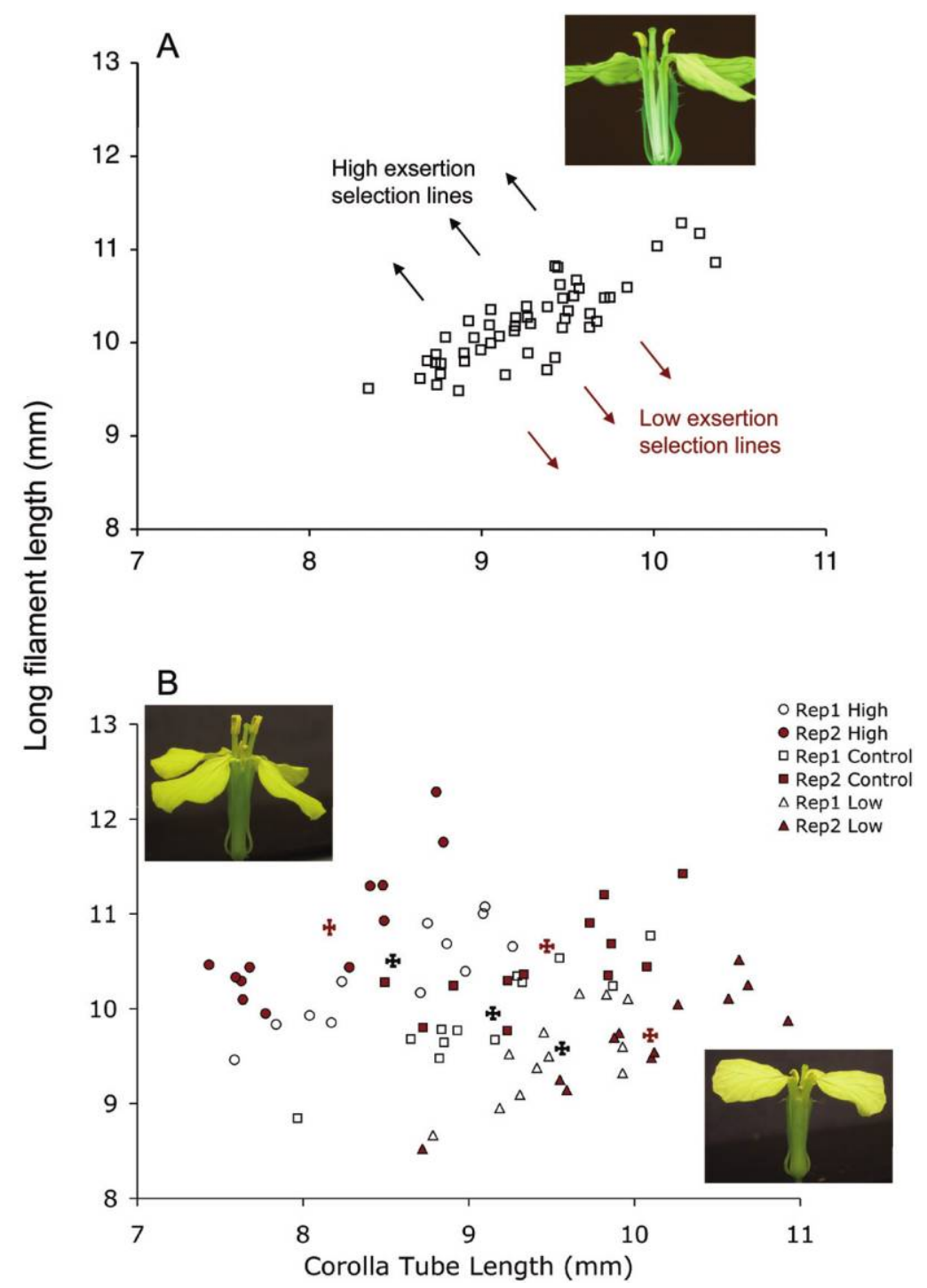

Figure 1: A, Half-sibling family means for filament and corolla tube lengths, depicting the additive genetic correlation for the natural population before selection (data from Conner and Via 1993). Arrows indicate the approximate directions of artificial selection applied to this population, with the black arrows pointing to the upper left representing the two high exsertion lines and the red arrows pointing to the lower right representing the two low exsertion lines. Because these plants were grown at a different time than those in $B$, the means for the two traits differed because of the different environmental conditions. To remove these mean differences while maintaining an unchanged correlation, the values in $A$ were adjusted to make the overall means the same as those for the control lines after selection by subtracting the mean difference in each trait from each value. $N=75 . B$, Full-sibling family means after eight (Rep 1$)$ or nine (Rep 2 ) generations of selection. Crosses represent the bivariate means $( \pm 1$ SEM) for each group. Total $N=72(12$ families per line $\times 2$ replicates per treatment $\times 3$ selection treatments); each point represents the mean value for 10 plants grown from each family.

variation in the parallel direction. However, the degree to which the response will be slowed does not depend on the value of the correlation itself but rather on the direction and magnitude of selection applied to each trait and the entire $\mathbf{G}$ matrix. Therefore, quantitative predictions must be made on a case-by-case basis, and this is perhaps why theoretical quantitative predictions of the magnitude of constraint caused by genetic correlations have been relatively uncommon (but see Via and Lande 1985).

The persistence of a correlational constraint over evolutionary time depends on the genetic mechanism underlying the correlation. Correlations caused by pleiotropy or tight linkage are much more likely to cause constraint than those caused by linkage disequilibrium between mod- 
erately or loosely linked loci. This is because correlations due to pleiotropy are likely to be much more stable over time than those due to linkage disequilibrium (Clark 1987; Futuyma 1998). In the absence of selection, linkage, and assortative mating, linkage disequilibrium is halved in each generation by recombination, and very tight linkage is necessary to slow this process substantially (Falconer and Mackay 1996). Correlations due to pleiotropy, on the other hand, persist in the absence of selection. The exceptions to this pattern are loci that are extremely tightly linked; linkage disequilibrium between loci that rarely recombine will be evolutionarily similar to single pleiotropic loci. Therefore, only genetic correlations due to pleiotropy or extremely tight linkage are stable enough to cause evolutionary constraints.

Artificial selection is a uniquely well-suited technique for the study of constraints, because the evolution of traits in response to known selection pressures can be directly quantified (Maynard Smith and Sondhi 1960; Maynard Smith et al. 1985; Barton and Partridge 2000). For correlated traits, artificial selection can be applied perpendicular to the major axis of the correlation, which corresponds to the direction of minimum genetic variance in bivariate space (fig. 3 in Conner 2003; see also fig. $1 A$ in this article). Selection in this direction should result in the slowest possible response and thus the maximal evolutionary constraint. Several studies using this basic idea have been performed (Bell and Burris 1973; Weber 1990; Dorn and Mitchell-Olds 1991; Wilkinson 1993; Stanton and Young 1994; Emlen 1996; Beldade et al. 2002; Frankino et al. 2005, 2007; Allen et al. 2008), and all but two (Dorn and Mitchell-Olds 1991; Allen et al. 2008) have shown that short-term evolution of correlated traits can occur perpendicular to the major axis of the correlation.

Therefore, there is little doubt that evolution can occur perpendicular to the major axis of a correlation as long as the magnitude of the correlation is $<1$, but there is also little theoretical doubt that this evolution will be slower than if there were no correlation or if the selection was along the major axis of the correlation. The open question is how evolutionarily important the constraint caused by genetic correlations is likely to be. One way to answer this question is through empirical estimates of the speed of evolution perpendicular to pleiotropic correlations of high magnitude, as only these are theoretically expected to cause strong constraint. Furthermore, to judge the importance of the constraint, these estimates should be made in a system for which natural selection on the correlated traits is well understood and the divergence in these traits among related species has been measured.

The correlation between the filament and corolla tube lengths in wild radish (Raphanus raphanistrum) flowers provides an ideal natural opportunity to test constraints.
The additive genetic correlation is very high and has been estimated with good precision (mean \pm SEM, $0.85 \pm$ 0.06; Conner and Via 1993), using a large nested halfsibling design (50 sires, 4 dams per sire, 1,133 total offspring). Unlike most correlations in natural populations, this correlation is known to be caused by pleiotropy or extremely tight linkage, as it was unchanged in two large replicate populations $\left(N_{\mathrm{e}}=600\right.$ each) subjected to nine generations of enforced random mating with relaxed selection (Conner 2002). Correlations caused by linkage disequilibrium would be altered by recombination under relaxed selection.

In addition, natural selection on this correlation and the composite trait determined by the relative lengths of filament and corolla tube, anther exsertion, have been well studied in wild radish. Anther exsertion is defined as filament length minus corolla tube length, and thus it describes the position of the anthers relative to the opening of the corolla tube (fig. 2). Measurements of selection based on lifetime seed-siring success by all pollinators showed significant stabilizing selection on anther exsertion in the first year, a combination of stabilizing and directional selection for an increase in the second year, and only directional selection for increased exsertion in the third year (Morgan and Conner 2001). Stabilizing selection on the difference between two traits is equivalent to correlational selection on these traits (Phillips and Arnold 1989; Brodie 1992); therefore, there was selection to increase the correlation in the first 2 years and to increase filament length relative to corolla tube length in the second and third years. Note that the directional selection on anther exsertion represents selection perpendicular to the major axis of the filament-corolla tube correlation, similar to that in the artificial selection experiments discussed above.

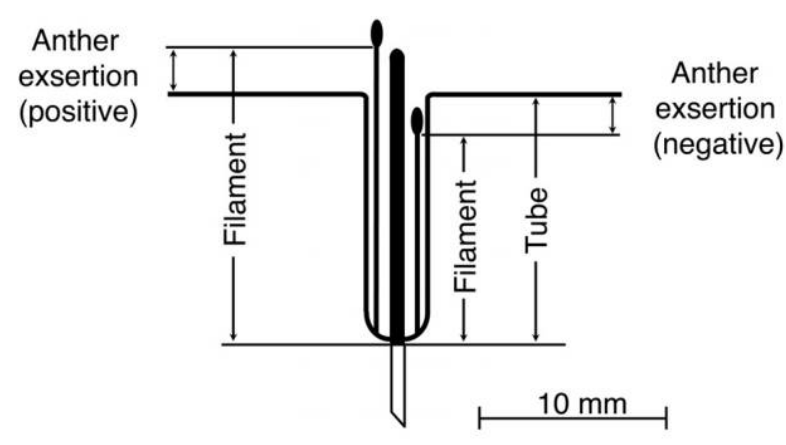

Figure 2: Lateral cross-section diagram of a wild radish flower, showing the traits examined in this study. Only two of the six filaments are shown for clarity. Anther exsertion is calculated as filament length minus tube length; examples of both positive and negative anther exsertion are shown. 
The differences in selection among years may be due to differences among pollinators in the selection they exert through differences in male fitness (seed-siring success). The most common pollinator genus of wild radish in the upper Midwest is the sweat bee Dialictus, which exerts stabilizing selection on anther exsertion, while two of the larger pollinators, honey bees and cabbage butterflies (Pieris rapae), exert directional selection on exsertion (Conner et al. 2009; H. F. Sahli and J. K. Conner, unpublished manuscript). The underlying functional basis for this selection has also been studied in wild radish. Visits by sweat bees have maximal pollen removal (a component of male fitness) with intermediate anther exsertion, while pollen removal by honey bees and cabbage butterflies increases with increasing exsertion (Conner et al. 1995, 2009).

A comparative study of filament and corolla tube lengths across the Brassicaceae suggests that intermediate anther exsertion is either constrained or adaptive across much of the family, as most species have roughly equal filament and corolla tube lengths, placing the anthers at the opening of the corolla tube (fig. 14.6 in Conner 2006). This fits with the stabilizing selection on exsertion exerted by sweat bees in wild radish. However, there is also comparative evidence for selection perpendicular to the major axis, as several species of Brassicaceae show highly exserted or highly inserted anthers (Conner 2006); the exserted species are consistent with the directional selection for increased exsertion exerted by butterflies and honey bees in wild radish.

In this study, we used artificial selection perpendicular to the major axis of the filament-corolla tube correlation in wild radish to directly test for an evolutionary constraint caused by this correlation. We also used new estimates of the $\mathbf{G}$ matrix for these traits from the selection lines to assess changes due to selection or drift and to make quantitative predictions of the evolutionary response to this artificial selection, enabling us to more fully assess the role of genetic correlations in causing constraints from both theoretical and empirical perspectives.

\section{Material and Methods}

\section{Artificial Selection}

To perform selection perpendicular to the major axis of the correlation between the filament and corolla tube lengths (fig. $1 A$ ), we conducted directional selection on an index trait, anther exsertion. Anther exsertion is defined as the difference between the long filament and corolla tube lengths. Because the selection imposed was linear, it is selection to change the bivariate mean. This form of selection is not correlational selection, which is equivalent to nonlinear selection on an index trait (Phillips and Arnold 1989; Brodie 1992). The initial base population of 477 plants was derived from the New York natural population (Conner and Via 1993) that had been maintained in large random-mating greenhouse populations $\left(N_{\mathrm{e}} \approx\right.$ 600) for seven generations (Conner 2002). Six selection lines were established from this base population, two were selected for increased exsertion (depicted by the arrows pointing to the upper left in fig $1 A$ ), two were selected for decreased exsertion (arrows pointing to the lower right in fig $1 A$ ), and two were random-mated controls. One replicate set of lines (one high exsertion, one low exsertion, one control) was selected for eight generations (rep 1), and the other set was selected for nine generations (rep 2 ). Generations 1-3 of both replicates were grown in the same greenhouse room at the University of Illinois, generations $4-5$ of rep 1 were grown in the same greenhouse room at Reed College in Oregon, and generations 4-9 of rep 2 and generations 6-8 of rep 1 were grown at Kellogg Biological Station (KBS) in Michigan. Within each generation and line, one to 10 offspring were successfully grown and measured from each of 12 families from the previous generation; a total of 4,970 plants were measured over the course of the study. One individual from each family was chosen for mating; individuals with the most extreme anther exsertion were chosen in the selection lines, and an individual was chosen at random in the control lines. This family selection design maximizes the effective population size and thus minimizes drift (Hill 1980). Chosen individuals were mated randomly so that every plant acted as a male and a female, but with no reciprocal crosses.

To test for a significant response to selection in anther exsertion and its component traits, filament and corolla tube lengths, a mixed-model analysis (Proc Mixed; SAS Institute 2004) was performed on the data from the last generation. High and low lines were compared with controls within each set of replicates that had been grown simultaneously in the same greenhouse room, with selection direction as a fixed effect and family nested within selection direction as a random effect.

To test for the linearity of the selection response, response values were regressed on the cumulative selection differentials using linear only and linear plus quadratic terms (Falconer and Mackay 1996). To remove the effects of environmental differences among generations in this analysis, values of both the response and the cumulative selection differentials in the high lines were subtracted from the low-line values within each replicate (Hill 1980). Because the pairs of high and low lines within each replicate were grown simultaneously in the same greenhouse room, they represent the total genetic response to selection 
in both directions after removing environmental differences, similar to a common-garden design.

To determine whether selection caused changes in genetic variance or covariance, the $\mathbf{G}$ matrix within each line was estimated using the phenotypic values for filament and corolla tube from the last four (rep 1) or five (rep 2) generations of pedigree information. These pedigrees include a mix of full- and half-sibling families, and the sample sizes within each of the lines were between 459 and 474 , so these estimates of $\mathbf{G}$ are reasonably precise.

\section{Predicted Responses to Selection}

We combined these estimates of the $\mathbf{G}$ matrix within each line with estimates of artificial selection gradients from this study to produce bivariate predictions of the response to our artificial selection. For each episode of selection, the relative fitness of all plants in the parental generation was regressed on the filament and corolla tube lengths to estimate the selection gradients for these traits. Relative fitness for each plant was calculated as the number of offspring successfully grown and measured in the next generation (which varied from one to 10 for the selected parents and was zero for all unselected individuals) divided by the average number of offspring per plant in that generation. Cumulative selection gradients were obtained by summing across generations; these were then combined with the $\mathbf{G}$ matrix to predict the selection response. A confidence region for this response was obtained via parametric bootstrap (Efron and Tibshirani 1994). To do this, 1,000 selection gradients and an equal number of $\mathbf{G}$ matrices were generated by resampling from the parametric distribution of each, as described by their dispersion matrices. The product of each pair of these was taken, yielding 1,000 new response predictions. We found the variance matrix of this cloud of points and used it to generate confidence ellipses (1.96 times the square root of the variance in all directions) around the predicted mean responses.

\section{Results}

Anther exsertion showed a rapid response to selection through all generations (fig. 3); this response to selection in anther exsertion was highly significant relative to controls in all four selection lines (table 1). When plotted as a divergence between the replicate high and low lines versus the cumulative selection differential, the response was quite linear, with little or no sign of a slowing of response, although there is weak evidence for slight curvature in the rep 2 plot (fig. 4). Examining the bivariate distributions of the two individual traits, filament and corolla tube lengths, at the end of the selection shows the strong re- sponses of the two traits perpendicular to the major axis of the correlation (fig. 1B). The distribution of the randomly mated controls matches that of the base population, and there is little overlap between the three treatment groups.

The response in anther exsertion was due to evolution of filament and corolla tube lengths in opposite directions (fig. $1 B$; table 1). In both replicates, these two components of anther exsertion were highly significantly different between the high and the low lines, and in all but one case (the long filament in the rep 2 high line), each component trait was at least marginally significantly different from controls (table 1).

There were no clear patterns of changes in the $\mathbf{G}$ matrix in the selected lines relative to controls. In rep 1, the first eigenvalue was smaller and the second was greater in both selection lines, compared with controls, leading to slightly decreased genetic correlations, but exactly the opposite pattern occurred in rep 2 (table 2). Thus, the predicted decrease in genetic variance due to selection along eigenvector 2 (the direction on which we selected) is not yet apparent, but note that these matrices are estimated using the last four generations, so there may be more reduction in variance in the last generation. The variances, covariances, and eigenvalues tended to be smaller in all six lines (including the controls) than in the original population (table 2; fig. 5), which is consistent with a similar loss of variance due to drift in all six lines; however, since the original population was grown at a different time, these results could be due to environmental differences in gene expression rather than actual genetic change. Also note that the orientation of the bivariate ellipse (i.e., the eigenvectors) has changed in the rep 2 high exsertion line.

In both replicates, the actual response to selection was more constrained than the predictions based on the strength of artificial selection and the G matrix, but in both cases the actual responses were inside the $95 \%$ confidence ellipse of the prediction (fig. 6). Thus, the rapid response we observed was predictable on the basis of standard quantitative genetic theory. Note that the magnitude of the predicted response and its confidence ellipse reflect the accumulated uncertainty from the estimation of several generations of selection gradients as well as uncertainty in G.

\section{Discussion}

Our results show that wild radish filament and corolla tube lengths evolved in different directions in response to selection perpendicular to the strong positive genetic correlation between these traits in fewer than 10 generations. This rapid evolution was in the direction of minimum genetic variance in bivariate space and was in spite of good 


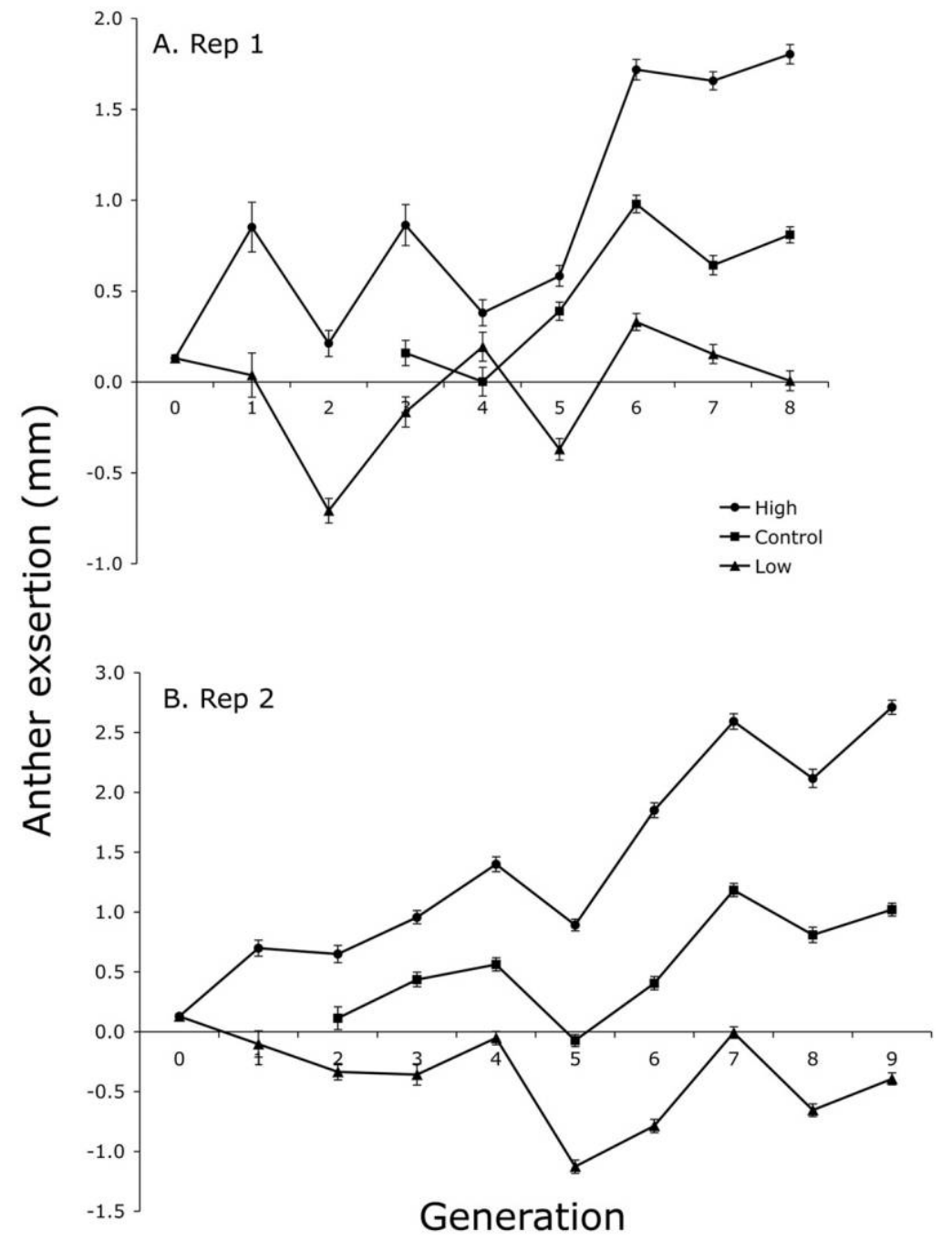

Figure 3: Mean response to selection on anther exsertion versus generation in each of the six lines ( \pm SEM). Traits were not measured in the control lines in the first two generations of the first replicate set of lines (rep 1) and the first generation of rep 2. The three rep 1 lines were not grown at the same time in generation 4, so the relative values of the means include both genetic and environmental differences; in all other cases the three lines within each replicate were grown simultaneously in the same greenhouse room, so differences among means within generation and replicate are caused by genetic differences only. Average $N$ for each mean is 93.6 ; total $N=4,970$ plants.

evidence for the strength and stability of this correlation, including that the genetic correlation was very high in magnitude, well estimated, and likely caused by pleiotropy (Conner 2002). The $\mathbf{G}$ matrix was also stable in response to the artificial selection imposed in this study. The repeatable and linear responses in the direction that we selected and the fact that both controls maintained the ancestral trait distribution strongly suggest that our selection was much stronger than the effects of drift.

To be convincing, our assertion of rapid evolution needs a null hypothesis for comparison; in other words, evolution of anther exsertion was rapid relative to what stan- dard? The inset photographs in figure $1 B$ show examples of anther exsertion resulting from the artificial selection, representing phenotypes not observed in tens of thousands of plants from the New York population raised in the field and greenhouse (J. K. Conner, personal observation). Our pairs of selection lines diverged 3.4 (rep 1) and 5.8 (rep 2) standard deviations in eight and nine generations, respectively (the standard deviation of anther exsertion was 0.53 in the base population); these are clearly biologically significant changes over a short period of time. The strongest standardized directional selection gradient on anther exsertion observed by Morgan and Conner (2001) was 
Table 1: Mean \pm SEM $(\mathrm{mm})$ differences in length between the filaments and the corolla tubes of the base population before selection and of the six artificial selection lines after eight (rep 1) or nine (rep 2) generations of selection on anther exsertion

\begin{tabular}{|c|c|c|c|c|c|c|c|c|c|}
\hline & \multicolumn{3}{|c|}{ Anther exsertion } & \multicolumn{3}{|c|}{ Long filament } & \multicolumn{3}{|c|}{ Corolla tube } \\
\hline & Mean \pm SEM & $F$ & $P$ & Mean \pm SEM & $F$ & $P$ & Mean \pm SEM & $F$ & $P$ \\
\hline Base & $.13 \pm .02$ & & & $9.80 \pm .05$ & & & $9.67 \pm .05$ & & \\
\hline \multicolumn{10}{|l|}{ Rep 1: } \\
\hline High 1 & $1.80 \pm .05$ & 106 & $<.0001$ & $10.36 \pm .09$ & 4.1 & .06 & $8.55 \pm .07$ & 5.9 & .02 \\
\hline Control 1 & $.81 \pm .04$ & & & $9.92 \pm .08$ & & & $9.11 \pm .08$ & & \\
\hline Low 1 & $.01 \pm .06$ & 52.4 & $<.0001$ & $9.51 \pm .08$ & 3.8 & .06 & $9.51 \pm .08$ & 4.2 & .05 \\
\hline High-low 1 & 1.79 & 230 & $<.0001$ & .85 & 16.9 & .0005 & -.96 & 25.2 & $<.0001$ \\
\hline \multicolumn{10}{|l|}{ Rep 2: } \\
\hline High 2 & $2.71 \pm .06$ & 113 & $<.0001$ & $10.80 \pm .09$ & 1.6 & .22 & $8.09 \pm .07$ & 39.3 & $<.0001$ \\
\hline Control 2 & $1.02 \pm .05$ & & & $10.48 \pm .07$ & & & $9.46 \pm .08$ & & \\
\hline Low 2 & $-.39 \pm .05$ & 101 & $<.0001$ & $9.68 \pm .08$ & 13.9 & .001 & $10.08 \pm .09$ & 6.6 & .02 \\
\hline High-low 2 & 3.10 & 554 & $<.0001$ & 1.12 & 18.3 & .0003 & -1.99 & 74.7 & $<.0001$ \\
\hline
\end{tabular}

Note: The three selection lines within each replicate were grown simultaneously but the base was grown before the selection started, so valid comparisons can be made only within each replicate because of environmental differences. The $F$ (degrees of freedom $=1,22$ for all) and $P$ values are from mixed-model comparisons of each selection line to the matching control (see "Material and Methods"), and the "high-low" rows give the mean difference between the high and the low lines within each replicate and the results of the mixed-model test for a difference between the high and low means. $N=477$ for base and $N=107-120$ for each selection line (the smaller SEM for the base is due to the larger sample size).

0.17, so the increase in exsertion of 3.2 standard deviations seen in high rep 2 compared with the control (see table 1) translates to a $54 \%$ increase in relative fitness (assuming that the selection gradient remained unchanged), which is also a biologically important change in just nine generations. Note that pure directional selection was observed in only one of 3 years; there was stabilizing selection on anther exsertion in the other 2 years (see "Introduction").

Quantitative genetic theory predicts that the rate of this evolution would have been much faster had we selected along the major axis of the correlation (e.g., Beldade et al. 2002) or if there had been no genetic correlation between these traits; however, given the speed of the response in our experiment, the evolutionary importance of this constraint is questionable. To make a rough prediction of what the response to the selection we imposed would have been without a correlation, we set the additive genetic covariance between filament and corolla tube to zero but used the estimated genetic variances for each trait in the natural population (table 1); this is related to approaches used by Smith and Rausher (2008) and Agrawal and Stinchcombe (2009). Multiplying this $2 \times 2$ G matrix by the cumulative selection gradients for the two traits, the predicted evolution of the filament and corolla tube lengths would have been five times faster (averaged across traits and replicates) than that predicted with the actual covariance. We can also roughly model evolution along the major axis of the correlation by making the selection gradients for both traits positive in all lines (instead of opposite signs, as the actual selection gradients in the ex- periment were) and using the actual estimated $\mathbf{G}$ matrix. In this case, the evolution would have been 11 times faster on average than that predicted on the basis of the actual gradients. This is even faster than the zero-covariance case because the response is augmented by the covariance along the major axis. Given that our predicted change was 1-3 $\mathrm{mm}$ with the correlation (open circles in fig. 6), the unconstrained and augmented rates of evolution are unlikely to persist more than a few generations given that the traits involved are only $\sim 10 \mathrm{~mm}$ long; either variance would be depleted or the population would reach a new optimum in nature.

The speed of our response to selection and the evolutionary importance of the constraint caused by the filament-corolla tube correlation can also be judged by comparison with related species. The evolution of increased and decreased anther exsertion without a change in the correlation between the filament and corolla tube component traits that we observed in our experiment has occurred repeatedly in Brassicaceae (Conner 2006). While most of the 24 species studied had filaments and corolla tubes of roughly equal length, as occurs in wild radish naturally, two of the sampled species have evolved positive exsertion (filaments longer than corolla tubes; Aethionema, Stanleya) and two have inserted anthers (filaments shorter than corolla tubes; Hesperis, Mattiola), in an exact parallel to the results of our artificial selection reported here. At the average rate of change seen in the high and low lines of rep 2, it would take only 36 generations for wild radish to evolve to the same exsertion (measured as the log dif- 


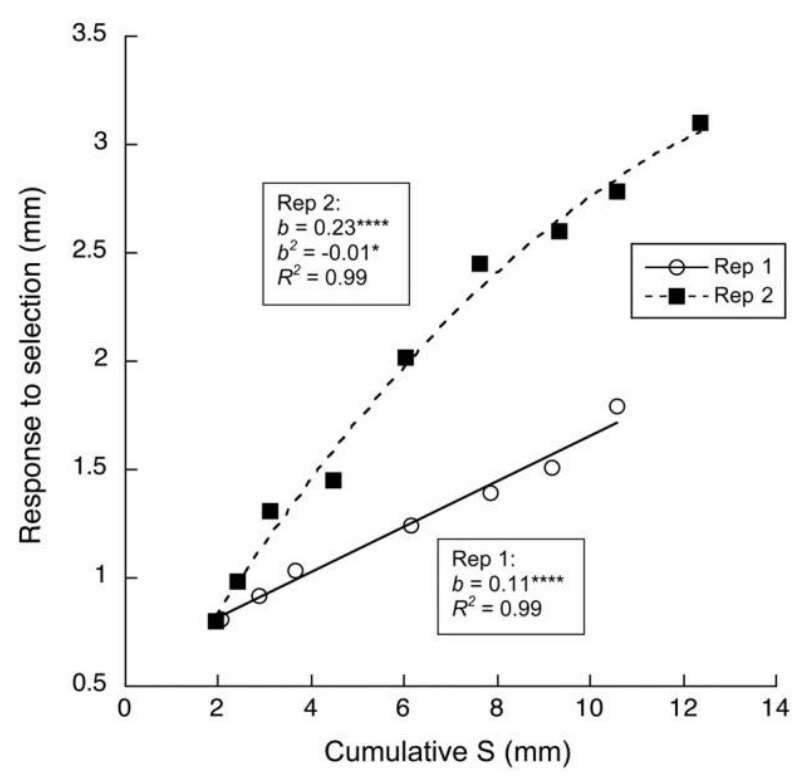

Figure 4: Results of the artificial selection experiment, plotted as the response of the composite trait (anther exsertion) regressed on cumulative selection differential. Each point represents the difference in anther exsertion between the high and the low lines and the absolute selection differential summed over both directions of selection for each generation and replicate. Because the high and the low lines within each replicate were grown simultaneously (with one exception noted below), differences in mean phenotype between them are due entirely to genetic change; by plotting these differences, phenotypic variation among generations that are caused by changes in the greenhouse environment over time are removed and the regressions reflect only true genetic evolution. For example, the last point for the second set of replicate lines (rep 2) shows that after nine generations, the anthers of the high lines were exserted $3.1 \mathrm{~mm}$ more than those of the low lines. The first set of replicate lines (rep 1) was selected for eight generations, and rep 2 for nine; data for generation 4 are not plotted for rep 1 because the high and the low lines were not grown simultaneously and therefore the environmental effects cannot be separated from genetic effects. $P$ values (one asterisk indicates $P<.05$; four asterisks indicates $P<.0001$ ) should be interpreted with caution, as the points are not independent. The quadratic term for rep 1 was not significant.

ference, to remove size effects) as the most exserted species (Stanleya) and about 81 generations to evolve to the same exsertion as the most inserted species (Mattiola). Note that each generation represents only 1 year for wild radish, an annual plant.

There are both theoretical and empirical reasons to believe that our results are not simply limited to this pair of traits in this species but are in fact more general. First, the prediction of evolution perpendicular to the major axis using standard theory (Lande 1979) parameterized by the observed $\mathbf{G}$ matrices and selection gradients imposed in our study makes it clear that rapid evolutionary change can occur despite a strong genetic correlation (fig. 6). Sec- ond, artificial selection on several species of insects and a wild population of the domestic radish has been able to independently change the means of a wide variety of correlated traits (Bell and Burris 1973; Weber 1990; Wilkinson 1993; Stanton and Young 1994; Emlen 1996; Beldade et al. 2002; Frankino et al. 2005, 2007; but see Allen et al. 2008); in other words, these studies were able to change the position of the bivariate cloud of points in spite of a correlation (cf. fig. 1B). While in none of these cases was the mechanism of correlation known, and in only one case was there a rigorous published estimate showing an additive genetic correlation that was high in magnitude (Frankino et al. 2007), it seems likely that the genetic correlations were at least moderate in magnitude and caused at least in part by pleiotropy, because the traits were mostly size related. In addition, two studies (Bell and Burris 1973; Frankino et al. 2007) reported an unchanged genetic correlation after selection, two others showed a relatively unchanged bivariate phenotypic distribution before and after selection (Emlen 1996; Frankino et al. 2005), and another two show a correlated phenotypic distribution after selection (Weber 1990; Wilkinson 1993); these results also suggest pleiotropy. Clearly, the similar results in both plants and animals for a variety of different kinds of traits strongly suggest that genetic correlations by themselves are insufficient to quantify the nature and severity of evolutionary constraint. Note that in a few of these cases (but not most) there is evidence for (Frankino et al. 2005) or at least a hypothesis of (Stanton and Young 1994; Frankino et al. 2007) the correlation being adaptive (as is true for the filament-corolla tube correlation in wild radish).

Therefore, evolution can be rapid despite genetic correlations of $<1$ (see Pigliucci and Kaplan 2000). Whether genetic correlations can produce evolutionarily significant constraints over the medium or long term or whether strong constraints due to lack of variance along some axis in multivariate space are common in nature (Blows and Hoffmann 2005; but see Mezey and Houle 2005) are open questions. It seems likely that the smaller amount of genetic variance along the minor axis means that this variance will be depleted more rapidly than would be true along the major axis, causing an earlier selection plateau in the former case. Also still open is the question of whether genetic correlations bias the course of interspecific evolution along "genetic lines of least resistance" (Schluter 1996). Species seem to usually diverge along the major axis of correlated traits, that is, the direction of maximum variance in bivariate space (Schluter 1996; Baker and Wilkinson 2003; Begin and Roff 2004; Marroig and Cheverud 2005; McGuigan et al. 2005; Conner 2006; Renaud et al. 2006), which could indicate either constraint or a ridge in the adaptive landscape (Schluter 1996). However, all of these studies found some species that deviate strongly from 
Table 2: Additive variances $\left(V_{\mathrm{A}}\right)$ and covariances ( $\operatorname{cov}_{\mathrm{A}}$; together comprising the $\mathbf{G}$ matrix) for corolla tube and long filament lengths, the two eigenvalues of this $\mathbf{G}$ matrix, and the additive genetic correlation $\left(r_{\mathrm{A}}\right)$ between these two traits in the natural population before selection and in the last four generations of the six selection lines

\begin{tabular}{lcccccc}
\hline & Tube $V_{\mathrm{A}}$ & $\operatorname{cov}_{\mathrm{A}}$ & Long filament $V_{\mathrm{A}}$ & Eigenvalue 1 & Eigenvalue 2 & $r_{\mathrm{A}}$ \\
\hline Natural & .56 & .49 & .65 & 1.10 & .11 & .81 \\
High 1 & .44 & .41 & .50 & .88 & .06 & .87 \\
Control 1 & .50 & .45 & .49 & .95 & .05 & .91 \\
Low 1 & .46 & .35 & .41 & .78 & .08 & .81 \\
High 2 & .37 & .42 & .65 & .95 & .06 & .86 \\
Control 2 & .46 & .37 & .44 & .83 & .08 & .83 \\
Low 2 & .64 & .51 & .49 & 1.08 & .05 & .91 \\
\hline
\end{tabular}

Note: The estimates for the natural population are from a nested half-sibling design with 50 sires and 1,133 total offspring (Conner and Via 1993).

the major axis, suggesting that if there are constraints, they can be overcome by selection or drift. This may well be a general pattern, as these studies considered a wide range of taxa: mammals, birds, fish, insects, and plants. Alternatively, the species that deviate could have experienced a transient reduction in the correlation at some point in their evolutionary history, due to a major mutation (Agrawal et al. 2001). Further evidence suggesting that genetic correlations do not necessarily cause long-term constraint comes from the sexual dimorphism literature. Genetic correlations between the same trait expressed in males and females are usually very high (Roff 1997, p. 247), but sexual dimorphism is common and often evolves rapidly (Badyaev 2002; Chenoweth et al. 2008) in spite of this high correlation. There is evidence for a negative correlation between the across-sex genetic correlation and the degree of sexual dimorphism, suggesting some constraint, but the relationship is very weak (Poissant et al. 2010). Note that these points rest on the assumption that the high correlations across sexes we see today existed in the past before sexual dimorphism evolved.

The evolution of filament and corolla tube lengths in opposite directions in spite of the strong positive pleiotropic genetic correlation could have been due to several non-mutually exclusive mechanisms. The most likely cause is that there are loci affecting each of the two traits independently (i.e., not pleiotropic). Because the additive genetic correlation is not 1 , there is some independent genetic variation for selection to act on. Another possible cause is allelic variation at underlying pleiotropic loci; if some alleles cause higher or lower exsertion, then our selection would have increased the frequency of these alleles in the high or low lines, respectively. A third possibility is modifier loci (epistasis): perhaps there are loci that affect the relative lengths of the filaments and corolla tubes produced by the pleiotropic loci (that is, the elevation of the bivariate ellipse in fig. 1) without changing the effect of these loci on the correlation itself. Finally, if some of the correlation is due to tightly linked loci rather than pleiotropy, then recombination between these loci could be partly responsible. However, since the correlation was unchanged in nine generations of random mating (Conner 2002) and this experiment lasted only eight or nine generations, it seems likely that recombination would have been a minor contributor at best to the selection response. We are currently conducting a quantitative trait locus mapping study on a cross between the selection lines to shed light on these possible mechanisms.

A great deal of interest and effort has been devoted recently to understanding the evolution of the $\mathbf{G}$ matrix, the matrix of additive genetic variances and covariances (reviewed in Steppan et al. 2002). Much of the interest in G matrix evolution has stemmed from a desire to understand whether the $\mathbf{G}$ matrix could cause long-term constraint. From the results to date it seems that there is certainly some stasis in the $\mathbf{G}$ matrix, at least within species, but changes in the $\mathbf{G}$ matrix are common, sometimes over fairly short timescales (Steppan et al. 2002). In the studies showing deviations from genetic lines of least resistance mentioned above, the lines of least resistance were based on estimates of genetic correlations in only one or none of the taxa in the study, and in no case was the $\mathbf{G}$ matrix estimated for taxa that diverged from the lines of least resistance. Thus, it is unclear whether G-matrix evolution is necessary for evolutionary deviations; evidence from our work suggests that it may not be, because our artificial selection caused deviations in the mean of the traits without a change in the underlying genetic correlations. If this is more generally true, and true over longer timescales and greater phenotypic divergences, then it may mean that the stability of the $\mathbf{G}$ matrix is not as relevant to evolutionary constraints as was once thought. Note that simulations show that selection perpendicular to genetic lines of least resistance, such as that imposed in this study, decreases the evolutionary stability of the $\mathbf{G}$ matrix (Jones et al. 2004). 

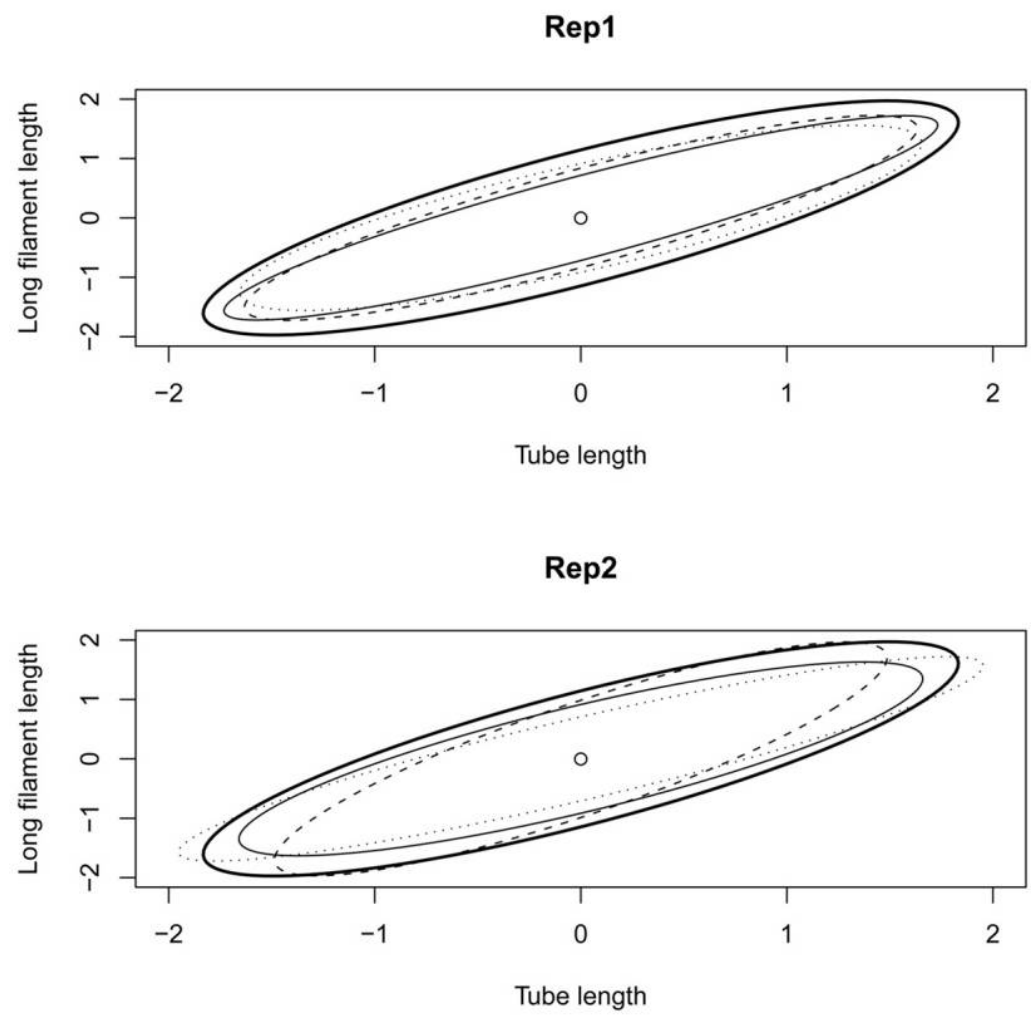

Figure 5: Graphical depictions of the bivariate $\mathbf{G}$ matrix in the base population (heavy solid line) and in the six selection lines. In each replicate, the high exsertion line is dashed, the low is dotted, and the control is represented by the thin solid line.

This discussion has focused on only complex quantitative traits that are likely affected by several to many loci. It is possible that constraint may be more likely in simple traits, because with fewer genes involved there may be more restrictions on possible evolutionary trajectories. Several recent studies have reported constraint in simple protein phenotypes (Miller et al. 2006; Weinreich et al. 2006; reviewed in Poelwijk et al. 2007), but these studies tested a fundamentally different kind of constraint than that tested in our study. Our study and the other artificial selection studies cited above have elucidated the magnitude of the constraint on the production of certain phenotypes, without regard to whether those phenotypes are adaptive in any particular environment. Indeed, the phenotypes produced by artificial selection perpendicular to the major axis of a correlation are often not adaptive (Frankino et al. 2005, 2007; J. K. Conner, K. Karoly, and H. F. Sahli, unpublished data). The protein phenotype articles cited above address whether evolution is constrained by the adaptive landscape, that is, whether certain changes are adaptive; thus, they test selective constraints sensu Arnold (1992). In other words, artificial selection studies (including this study) address how rapidly a given phe- notype can be produced, regardless of whether it is adaptive, while protein evolution studies address whether a given phenotype is adaptive when it is produced.

There are a number of unanswered questions concerning the role of genetic correlations as constraints on adaptive evolution. For example, how common are directions in multivariate space that clearly lack variance (Blows and Hoffmann 2005)? Is evolution in these directions strongly constrained, and if so, how often does selection act in these directions? It seems intuitive that correlations that are based on fundamental resource trade-offs might more strongly constrain evolution, but these kinds of constraints have rarely been directly tested by artificial selection perpendicular to the major axis of the correlation. Most or all of the studies (including this one) that have selected perpendicular to a correlation have used traits that were positively correlated dimensions, so therefore these traits were unlikely to be involved in a fundamental trade-off. An exception to this is the selection for earlier flowering at greater height in Brassica (Dorn and Mitchell-Olds 1991), to which there was a response to one generation of selection, although the gain in height was modest. The genetic correlation between these traits was also modest 


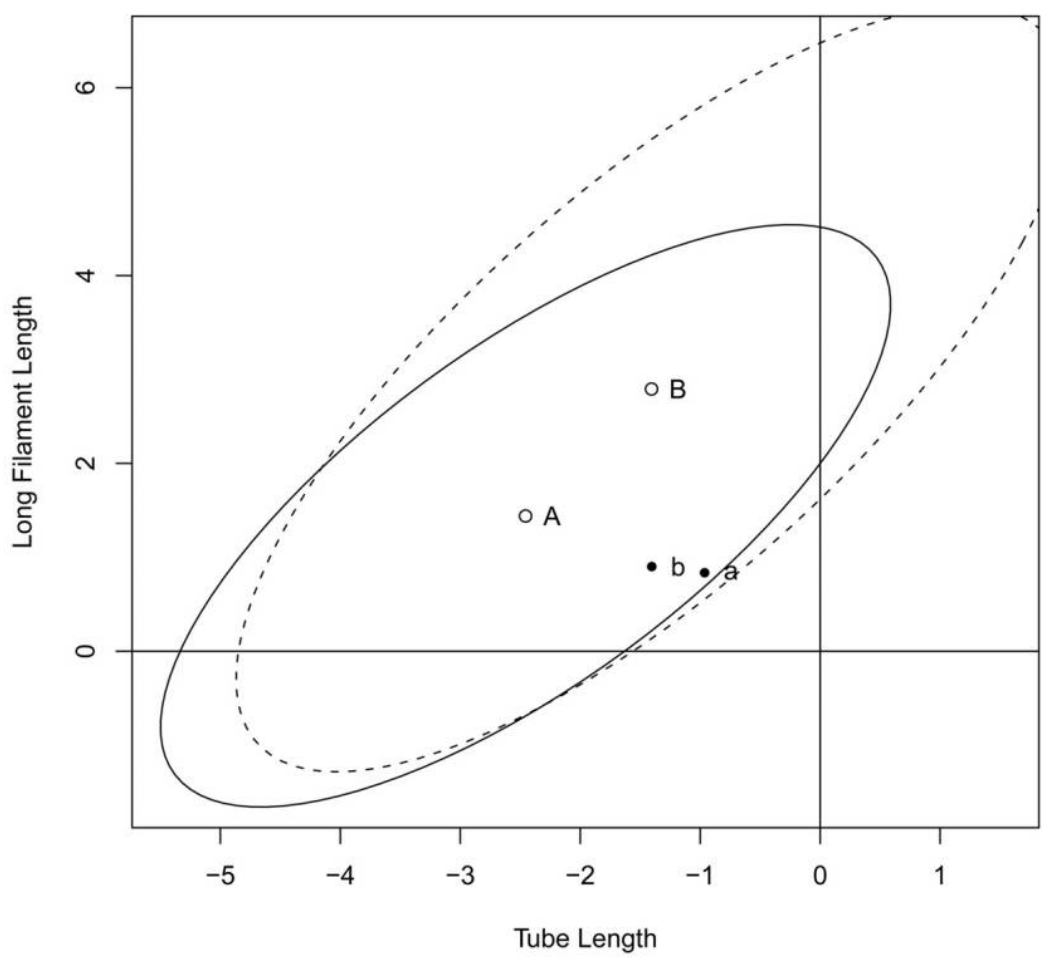

Figure 6: Predicted and actual responses to selection on filament and corolla tube lengths, with $95 \%$ confidence regions (from a parametric bootstrap; see "Material and Methods"), presented as the high selection line minus the low selection line (as in fig. 4). The open circles with uppercase letters are the predicted mean responses (centers of the ellipses) and the filled circles with lowercase letters are the actual responses. The first set of replicate lines (replicate 1) is the pair of points labeled $A$ with the solid ellipse, and replicate 2 is the pair of points labeled B with the dashed ellipse. Thus, in replicate 1, the high line was predicted to have long filaments $1.4 \mathrm{~mm}$ longer than those of the low line and corolla tubes $2.4 \mathrm{~mm}$ shorter, while the actual change was as follows: long filaments, $0.85 \mathrm{~mm}$ longer; corolla tubes, $0.96 \mathrm{~mm}$ shorter.

(0.61), so it would be very interesting to see how these traits would respond to more generations of selection. Unfortunately, many traits that we expect to be related by fundamental trade-offs do not have consistently negative genetic correlations (e.g., flower size and number; Houle 1991; Worley and Barrett 2000), so quantitative genetic models certainly would not predict a strong constraint. It may be that the best way forward is to determine the individual loci underlying trade-offs and genetic correlations and study their effects and evolution (i.e., change: in allele frequency) directly (Fry 1993).

\section{Acknowledgments}

We thank T. Hardway for initiating the selection lines, T. Hickox and many undergraduates for assistance in the lab and greenhouse, and M. Duffy, F. Knapczyk, C. Mills, A $\rightarrow$ Roles, and several anonymous reviewers for comments on the manuscript. This work was supported by grants from the National Science Foundation (DEB-9903880 to J.K.C. and K.K.) and the Cooperative State Research, Education, and Extension Service, U.S. Department of Agriculture (agreement no. $2002^{*} 35320-11538$ (to J.K.C.). This is KBS contribution 1591.

\section{Literature Cited}

Agrawal, A. F., and J. R. Stinchcombe. 2009. How much do genetic covariances alter the rate of adaptation? Proceedings of the Royal Society B: Biological Sciences 276:1183-1191.

Agrawal, A. F., E. D. Brodie III, and L. H. Rieseberg. 2001. Possible consequences of genes of major effect: transient changes in the Gmatrix. Genetica 112-113:33-43.

$\rightarrow$ Allen, C., P. Beldade, B. Zwaan, and P. Brakefield. 2008. Differences in the selection response of serially repeated color pattern characters: standing variation, development, and evolution. BMC Evolutionary Biology 8:94.

Antonovics, J., and P. H. van Tienderen. 1991. Ontoecogenophyloconstraints? the chaos of constraint terminology. Trends in Ecology \& Evolution 6:166-168. 


\section{The American Naturalist}

Arnold, S. J. 1992. Constraints on phenotypic evolution. American Naturalist 140(suppl.):S85-S107.

$\rightarrow$ Ashman, T. L., and C. J. Majetic. 2006. Genetic constraints on flora $\rightarrow$ evolution: a review and evaluation of patterns. Heredity 96:343352.

$\rightarrow$ Badyaev, A. V. 2002. Growing apart: an ontogenetic perspective on the evolution of sexual size dimorphism. Trends in Ecology $8 \rightarrow$ Evolution 17:369-378.

$\rightarrow$ Baker, R. H., and G. S. Wilkinson. 2003. Phylogenetic analysis of correlation structure in stalk-eyed flies (Diasemopsis, Diopsidae) $\rightarrow$ Evolution 57:87-103.

$\rightarrow$ Barton, N., and L. Partridge. 2000. Limits to natural selection. Bioessays 22:1075-1084.

Begin, M., and D. A. Roff. 2003. The constancy of the $\mathbf{G}$ matrix through species divergence and the effects of quantitative genetic constraints on phenotypic evolution: a case study in crickets. Evolution 57:1107-1120.

$\rightarrow \longrightarrow$. 2004. From micro- to macroevolution through quantitativı genetic variation: positive evidence from field crickets. Evolution 58:2287-2304.

$\rightarrow$ Beldade, P., K. Koops, and P. M. Brakefield. 2002. Developmenta' constraints versus flexibility in morphological evolution. Nature 416:844-847.

$\rightarrow$ Bell, A. E., and M. J. Burris. 1973. Simultaneous selection for two correlated traits in Tribolium. Genetics Research (Cambridge) 21 . $29-46$.

$\rightarrow$ Berenbaum, M. R., A. R. Zangerl, and J. K. Nitao. 1986. Constraints on chemical coevolution: wild parsnips and the parsnip webworm Evolution 40:1215-1228.

$\rightarrow$ Björklund, M. 1996. The importance of evolutionary constraints ir ecological time scales. Evolutionary Ecology 10:423-431.

$\rightarrow$ Blows, M. W., and A. A. Hoffmann. 2005. A reassessment of genetic limits to evolutionary change. Ecology 86:1371-1384.

$\rightarrow$ Brodie, E. D., III. 1992. Correlational selection for color pattern and antipredator behavior in the garter snake Thamnophis ordinoides. Evolution 46:1284-1298.

$\rightarrow$ Caruso, C. M., H. Maherali, A. Mikulyuk, K. Carlson, and R. B. Jackson. 2005. Genetic variance and covariance for physiological traits in Lobelia: are there constraints on adaptive evolution? Evolution 59:826-837.

$\rightarrow$ Chenoweth, S. F., H. D. Rundle, and M. W. Blows. 2008. Genetic constraints and the evolution of display trait sexual dimorphism ${ }_{1}$ by natural and sexual selection. American Naturalist 171:22-34.

Clark, A. G. 1987. Genetic correlations: the quantitative genetics of evolutionary constraints. Pages $25-45$ in V. Loeschcke, ed. Genetic $\rightarrow$ constraints on adaptive evolution. Springer, Berlin.

$\rightarrow$ Conner, J., and S. Via. 1992. Natural selection on body size in Tribolium: possible genetic constraints on adaptive evolution. Heredity 69:73-83.

$\rightarrow$. 1993. Patterns of phenotypic and genetic correlations among morphological and life history traits in wild radish, Raphanus raphanistrum. Evolution 47:704-711.

$\rightarrow$ Conner, J. K. 2002. Genetic mechanisms of floral trait correlation. in a natural population. Nature 420:407-410.

$\rightarrow-$ 2003. Artificial selection: a powerful tool for ecologists. Ecology $84: 1650-1660$.

. 2006. Ecological genetics of floral evolution. Pages 260-277 in L. D. Harder and S. C. H. Barrett, eds. Ecology and evolution of flowers. Oxford University Press, New York.

$\rightarrow$ Conner, J. K., R. Davis, and S. Rush. 1995. The effect of wild radish floral morphology on pollination efficiency by four taxa of pollinators. Oecologia (Berlin) 104:234-245.

Conner, J. K., H. F. Sahli, and K. Karoly. 2009. Tests of adaptation: functional studies of pollen removal and estimates of natural selection on anther position in wild radish. Annals of Botany 103: $1547-1556$.

Delph, L. F., J. L. Gehring, F. M. Frey, A. M. Arntz, and M. Levri. 2004. Genetic constraints on floral evolution in a sexually dimorphic plant revealed by artificial selection. Evolution 58:1936-1946.

Dickerson, G. E. 1955. Genetic slippage in response to selection for multiple objectives. Cold Spring Harbor Symposia on Quantitative Biology 20:213-224.

$\rightarrow$ Dorn, L. A., and T. Mitchell-Olds. 1991. Genetics of Brassica campestris. 1. Genetic constraints on evolution of life-history characters. Evolution 45:371-379.

Efron, B., and R. Tibshirani. 1994. An introduction to the bootstrap. Chapman \& Hall, New York.

Emlen, D. J. 1996. Artificial selection on horn length-body size allometry in the horned beetle Onthophagus acuminatus (Coleoptera: Scarabaeidae). Evolution 50:1219-1230.

Etterson, J. R., and R. G. Shaw. 2001. Constraint to adaptive evolution in response to global warming. Science 294:151-154.

Falconer, D. S., and T. F. C. Mackay. 1996. Introduction to quantitative genetics. Longman, Harlow.

Frankino, W. A., B. J. Zwaan, D. L. Stern, and P. M. Brakefield. 2005. Natural selection and developmental constraints in the evolution of allometries. Science 307:718-720.

$\rightarrow-2007$. Internal and external constraints in the evolution of morphological allometries in a butterfly. Evolution 61:2958-2970.

$\rightarrow$ Fry, J. D. 1993. The "general vigor" problem: can antagonistic pleiotropy be detected when genetic covariances are positive? Evolution 47:327-333.

Futuyma, D. J. 1998. Evolutionary biology. Sinauer, Sunderland, MA. $\rightarrow$ 2010. Evolutionary constraint and ecological consequences. Evolution 64:1865-1884.

Futuyma, D. J., M. C. Keese, and D. J. Funk. 1995. Genetic constraints on macroevolution: the evolution of host affiliation in the leaf beetle genus Ophraella. Evolution 49:797-809.

$\rightarrow$ Getty, T. 2000. A constrained view of constraints. Trends in Ecology \& Evolution 15:249.

Gould, S. J. 1989. A developmental constraint in Cerion, with comments on the definition and interpretation of constraint in evolution. Evolution 43:516-539.

Gould, S. J., and R. C. Lewontin. 1979. The spandrels of San Marco and the Panglossian paradigm: a critique of the adaptationist programme. Proceedings of the Royal Society B: Biological Sciences 205:581-598.

Hill, W. G. 1980. Design of quantitative genetic selection experiments. Pages 1-13 in A. Robertson, ed. Selection experiments in laboratory and domestic animals. Commonwealth Agricultural Bureau, Slough.

$\rightarrow$ Houle, D. 1991. Genetic covariance of fitness correlates: what genetic correlations are made of and why it matters. Evolution 45:630 648 .

$\rightarrow$ Jones, A. G., S. J. Arnold, and R. Burger. 2004. Evolution and stability of the G-matrix on a landscape with a moving optimum. Evolution 58:1639-1654.

$\rightarrow$ Lande, R. 1976. Natural selection and random genetic drift in phenotypic evolution. Evolution 30:314-334. 
$\rightarrow$ applied to brain : body size allometry. Evolution 33:402-416.

$\rightarrow$ Marroig, G., and J. M. Cheverud. 2005. Size as a line of least evolutionary resistance: diet and adaptive morphological radiation in new world monkeys. Evolution 59:1128-1142.

$\rightarrow$ Maynard Smith, J., R. Burian, S. Kauffman, P. Alberch, J. Campbell, B. Goodwin, R. Lande, D. Raup, and L. Wolpert. 1985. Developmental constraints in evolution. Quarterly Review of Biology 60:265-287.

Maynard Smith, J. M., and K. C. Sondhi. 1960. The genetics of a pattern. Genetics 45:1039-1050.

$\rightarrow$ McGuigan, K., S. F. Chenoweth, and M. W. Blows. 2005. Phenotypic divergence along lines of genetic variance. American Naturalis. 165:32-43.

$\rightarrow$ Mezey, J. G., and D. Houle. 2005. The dimensionality of genetic variation for wing shape in Drosophila melanogaster. Evolution 59: $1027-1038$

$\rightarrow$ Miller, S. P., M. Lunzer, and A. M. Dean. 2006. Direct demonstratiorı of an adaptive constraint. Science 314:458-461.

$\rightarrow$ Mitchell-Olds, T. 1996. Genetic constraints on life-history evolution: quantitative-trait loci influencing growth and flowering in Arabidopsis thaliana. Evolution 50:140-145.

$\rightarrow$ Morgan, M. T., and J. K. Conner. 2001. Using genetic markers to directly estimate male selection gradients. Evolution 55:272-281.

$\rightarrow$ O'Neil, P., and J. Schmitt. 1993. Genetic constraints on the independent evolution of male and female reproductive characters in the tristylous plant Lythrum salicaria. Evolution 47:1457-1471.

$\rightarrow$ Pease, C. M., and J. J. Bull. 1988. A critique of methods for measuring life history trade-offs. Journal of Evolutionary Biology 1:293-303.

$\rightarrow$ Phillips, P. C., and S. J. Arnold. 1989. Visualizing multivariate selection. Evolution 43:1209-1222.

$\rightarrow$ Pigliucci, M., and J. Kaplan. 2000. The fall and rise of Dr. Pangloss: adaptationism and the Spandrels paper 20 years later. Trends ir Ecology \& Evolution 15:66-70.

$\rightarrow$ Pilson, D. 1996. Two herbivores and constraints on selection for resistance in Brassica rapa. Evolution 50:1492-1500.

$\rightarrow$ Poelwijk, F. J., D. J. Kiviet, D. M. Weinreich, and S. J. Tans. 2007. Empirical fitness landscapes reveal accessible evolutionary paths. Nature 445:383-386.
Poissant, J., A. J. Wilson, and D. W. Coltman. 2010. Sex-specific genetic variance and the evolution of sexual dimorphism: a systematic review of cross-sex genetic correlations. Evolution 64:97107.

$\rightarrow$ Renaud, S., J. C. Auffray, and J. Michaux. 2006. Conserved phenotypic variation patterns, evolution along lines of least resistance, and departure due to selection in fossil rodents. Evolution 60: 1701-1717.

Roff, D. A. 1997. Evolutionary quantitative genetics. Chapman \& Hall, New York.

SAS Institute. 2004. Base SAS 9.1 procedures guide. SAS Institute, Cary, NC.

$\rightarrow$ Schluter, D. 1996. Adaptive radiation along genetic lines of least resistance. Evolution 50:1766-1774.

$\rightarrow$ Smith, R. A., and M. D. Rausher. 2008. Selection for character displacement is constrained by the genetic architecture of floral traits in the ivyleaf morning glory. Evolution 62:2829-2841.

$\rightarrow$ Stanton, M., and H. J. Young. 1994. Selecting for floral character associations in wild radish, Raphanus sativus L. Journal of Evolutionary Biology 7:271-285.

$\rightarrow$ Steppan, S. J., P. C. Phillips, and D. Houle. 2002. Comparative quantitative genetics: evolution of the $\mathbf{G}$ matrix. Trends in Ecology \& Evolution 17:320-327.

$\rightarrow$ Via, S., and R. Lande. 1985. Genotype-environment interaction and the evolution of phenotypic plasticity. Evolution 39:505-522.

Weber, K. E. 1990. Selection on wing allometry in Drosophila melanogaster. Genetics 126:975-989.

$\rightarrow$ Weinreich, D. M., N. F. Delaney, M. A. DePristo, and D. L. Hartl. 2006. Darwinian evolution can follow only very few mutational paths to fitter proteins. Science 312:111-114.

Wilkinson, G. S. 1993. Artificial sexual selection alters allometry in the stalk-eyed fly Cyrtodiopsis dalmanni (Diptera, Diopsidae). Genetics Research (Cambridge) 62:213-222.

$\rightarrow$ Worley, A. C., and S. C. H. Barrett. 2000. Evolution of floral display in Eichhornia paniculata (Pontederiaceae): direct and correlated responses to selection on flower size and number. Evolution 54: 1533-1545.

Associate Editor: Edmund D. Brodie III Editor: Mark A. McPeek 